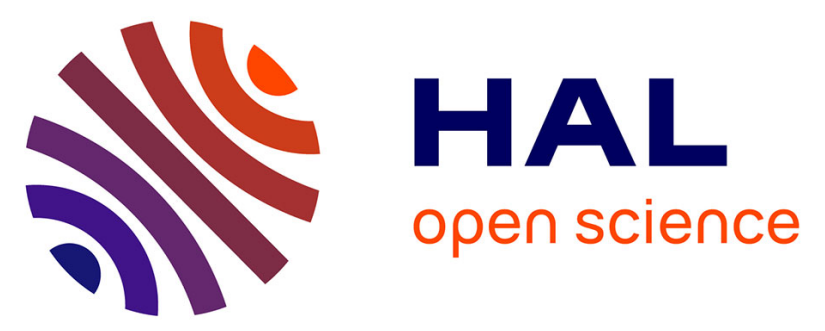

\title{
Relative importance of the functional abilities comprising Health Assessment Questionnaire Disability Index among rheumatoid arthritis patients
}

Risto Tuominen, Timo Möttönen, Carita Suominen, Tero Vahlberg, Sini Tuominen

\section{To cite this version:}

Risto Tuominen, Timo Möttönen, Carita Suominen, Tero Vahlberg, Sini Tuominen. Relative importance of the functional abilities comprising Health Assessment Questionnaire Disability Index among rheumatoid arthritis patients. Rheumatology International, 2009, 30 (11), pp.1477-1482. 10.1007/s00296-009-1176-0 . hal-00562262

\section{HAL Id: hal-00562262 \\ https://hal.science/hal-00562262}

Submitted on 3 Feb 2011

HAL is a multi-disciplinary open access archive for the deposit and dissemination of scientific research documents, whether they are published or not. The documents may come from teaching and research institutions in France or abroad, or from public or private research centers.
L'archive ouverte pluridisciplinaire HAL, est destinée au dépôt et à la diffusion de documents scientifiques de niveau recherche, publiés ou non, émanant des établissements d'enseignement et de recherche français ou étrangers, des laboratoires publics ou privés. 
Relative importance of the functional abilities comprising Health Assessment Questionnaire Disability Index among rheumatoid arthritis patients

Tuominen Risto, PhD (1), Möttönen Timo, MD (2), Suominen Carita, MD (1,3), Vahlberg Tero, MSc (1), Tuominen Sini, MStud (4)

(1) Department of Public Health, University of Turku

(2) Department of Internal Medicine, Rheumatology Unit, Turku University Hospital and University of Turku

(3) Department of Rehabilitation Medicine, Turku University Hospital and University of Turku

(4) Department of Internal Medicine, Rheumatology Unit, University of Kuopio

Correspondence and reprint requests:

Risto Tuominen

Department of Public Health , 20014 University of Turku, Finland

phone: +358 50585 2535, fax: +35823338439

e-mail: risto.tuominen@utu.fi 
Abstract

Objectives: To evaluate the relative importance of the twenty functions assessed by patients to produce the Health Assessment Questionnaire's Disability Index (HAQ-DI).

Methods: A systematic sample of 242 rheumatoid arthritis patients was drawn. Altogether 179 subjects returned acceptably filled questionnaire including perceived rheumatoid arthritis status in the 20 functions comprising the HAQ-DI. A separate complementary questionnaire was sent to 80 randomly chosen subjects. They were asked to estimate how important each of the same twenty items were in their everyday life by using the VAS method. Completely answered complementary questionnaires were returned by $66(88 \%)$ of the subsample.

Results: The relative importance of the functions assessed varied considerably. Men assessed the highest importance to the ability to walk outdoors on flat ground and women to dressing themselves, getting in and out of bed and washing and drying their body. For both genders the least important function was the ability to take a tub bath. The importance of all other functions except taking a tub bath were positively highly significantly correlated with the importance of each of the other functions. Factor analyses with one factor model showed high loadings on most functions, with the exception of 'Take a tub bath'. The two factor model formed one factor with loadings concentrating slightly more on 'lower extremity' and another on 'upper extremity'.

Conclusions: Assessment of RA patients' functional status would benefit from further development of measures by giving relative weights from patients' perspective to those functional abilities which are used for index computations.

Key words: Perception, function, disability, rheumatoid arthritis 
Introduction

Severity of rheumatoid arthritis is commonly studied by using numbers of painful or swollen joints [1], severity of pain [2], global health indices [3], and by assessing clinical disease activity [4-6] or functional ability $[7,8]$.

The most commonly used index to measure the functional ability and its limitations among RA patients has been Health Assessment Questionnaire Disability Index (HAQ-DI) [7, 8]. It comprises 20 functions which form eight domains [7]. A modified form of HAQ (MHAQ) was published by Pincus et al. in 1983 [9], where the number of functions was limited to eight, one question from each of the eight domains. However, both in the original HAQ-DI and in the MHAQ the selection of functions and their descriptions were based on professionals' intuitive choice $[7,9,10]$. Later studies have shown that disability items spontaneously generated by patients are different from those chosen by rheumatology health professionals [11-15]. The use of patient preferences has been suggested to supplement professionally chosen indicators $[15,16]$.

The many functions measured to produce the overall index figures representing the patients' functional status are given equal weight in most index computations [7-12, 16]. Although the use of equal weight makes the index figure computation easier, there is no scientific evidence to support the underlying idea that all the functions are equally important when determining a patient's functional status. This holds both for clinical assessment by health professionals and for patient perception.

The Aim 
The aim of this study was to evaluate the relative importance of the twenty functions assessed by patients to produce the Health Assessment Questionnaire's Disability Index.

Material and methods

A systematic sampling from the patient registers of the Hospital District of Southwest Finland was done. From ten consecutive calendar months (March-December 2006) the first twenty patients with diagnose M05.8 (Arthritis Rheumatoides seropositiva) and ten patients who had been diagnosed as having M06.0 (Arthritis Rheumatoides seronegativa) formed the basic sample. The proportions of seropositive and seronegative patients was known to be approximately $2: 1$. The patient registers include RA patients treated by rheumatologists of Turku University Hospital. The patients with the mildest disease course are mostly treated in primary health care and are thus underrepresented in our sample. Anticipated number of patients with these diagnoses were not available for each calendar month, and the final sample comprised 242 patients, $59.3 \%$ with M05.8 diagnosis.

The ethical committee of the Hospital District of Southwest Finland had approved the study, and the sampled subjects received a written description of the sampling, study purposes as well as the planned use and storage of the information they were to provide. This was followed by a description of the subject's rights according to the Helsinki declaration. The participating subjects were asked to give their written informed consent and written permission to collect data from their patient registers.

In the first phase all 242 subjects were sent a questionnaire form, including the above mentioned descriptions and permissions, together with a prepaid return envelope. Before the actual study questions, example questions with answering models were presented to make the answering task 
easier. The first part of the study questions covered socio-economic background. For this study the following socio-economic variables were formed: age in years, gender, number of persons in the same household analysed as living alone or with someone, level of income calculated as household disposable monthly income per person in the household, and retirement status.

After these general well being and experience of pain in general during the last week were asked by using 10 centimetre long double anchored Visual Analog Scales (VAS), with endpoints from worst possible (0) to best possible (100). The patient's assessments of numbers of swollen and painful joints were solicited.

Following questions dealt with the twenty items included to calculate the HAQ disability index. The subject was asked to estimate her/his ability to perform the given function during the last week using the 10 centimetre long double anchored VAS, with endpoints from 'could not at all' (0) to 'could without any problems' (100). The Finnish version of HAQ Disability Index has some expressions which differ from the American version. Instead of asking the ability to shampoo ones hair, the Finnish version asks the ability to wash ones hair. Opening a new milk cartoon is replaced by asking the ability to prepare ones own food, and doing chores such as vacuuming or yardwork is replaced by simply asking the ability to vacuum.

Altogether 179 subjects returned acceptably filled questionnaires (73.9\%). Detailed descriptions of sampling and the final sample characteristics have been reported earlier [15]. A separate systematic sample of 80 subjects was drawn from the respondents and a separate complementary questionnaire was sent to them. In this questionnaire the subjects were asked to estimate how important each of the same twenty items were in their everyday life. The estimates were solicited by using the 10 centimetre long double anchored VAS with endpoints from 'extremely unimportant' $(0)$ to 
'extremely important' (100). Completely answered complementary questionnaires were returned by $66(88 \%)$ of the subsample.

The distributions of the perceived importance scores were skewed to the left, with the exception of taking a tub bath which was skewed to the right. The Mann-Whitney U-test was used to compare the difference in perceived importance of the functions between genders. The Wilcoxon Signed Rank test was used to estimate the differences between importance scores. The associations between continuous variables were ascertained with Spearman's rank correlation coefficients. Exploratory factor analyses were performed for perceived importance functions with the maximum likelihood estimation method and oblimin-rotation. The correlation matrix used in factor analysis was computed using Spearman correlations coefficients due to skewed distributions. Statistical analyses were done using SPSS version 16.0 (SPSS Inc., Chicago, Illinois) and SAS Systems for Windows, release 9.1 (SAS Institute Inc., Cary, NC). P-values less than 0.05 were considered statistically significant.

Results

Overall, the relative importance of the functions assessed varied considerably. The subjects' socioeconomic background, general well-being and experience of pain or joint swelling did not affect the perceived importance. Men assessed the highest importance to the ability to walk outdoors on flat ground (97.0) and women to dressing themselves, getting in and out of bed and washing and drying their body (95.0). The least important function was the ability to take a tub bath both among men (6.5) and women (4.0). The differences between sexes were small (NS). (Table 1) 
In seven out of the eight domains used for computing the traditional HAQ, the most important function was the one which had been selected for MHAQ to represent that domain. However, in the Hygiene domain the difference in importance between the most important function 'Getting on and off the toilet' and the one chosen for MHAQ 'Wash and dry your body' was unsubstantial (Table 1).

Perceived importance varied more between subjects than within subjects. Those who expressed higher importance in one function, usually considered also other functions important. The importance of all other functions except taking a tub bath were positively highly significantly $(\mathrm{p}<0.01)$ correlated $(\mathrm{r}=0.324-0.878)$ with the importance of each of the other functions. Also subjects' perceived functional status varied considerably and a significant $(\mathrm{p}<0.05)$ positive correlation between perceived importance and functional ability of the same functions were observed only in cutting ones own meat $(r=0.257)$, vacuuming $(r=0.276)$ and getting in and out of $\operatorname{a~car}(r=0.271)$

Factor analyses with one factor model showed high loadings on most functions, with the exception of 'Take a tub bath', which had a very low loading. The two factor model formed one factor with loadings concentrating slightly more on 'lower extremity' and another on 'upper extremity'. However, the two-factor solution did not provide a unique solution, because five functions loaded (loadings $>0.30$ ) on both factors. Forcing more than two factors into the models did not provide any additional information. When evaluating the factor loadings within the eight domains forming the HAQ-DI score, practically all eight domains included more than one function with high loadings. However, the highest loadings within the domains were constantly observed for those eight functions which represent each domain in MHAQ computations except the difference in the Hygiene domain (Table 2). 


\section{Discussion}

The Hospital District of Southwest Finland provides health care services for approximately 460000 people residing in the area. It is assumed that the sampled patients are not selected, but the sample includes patients with varying lengths of disease history and in varying stages of RA at same proportions as in the RA patient population treated by rheumatologists [15]. However, one should be cautious when generalising the findings of this study to all RA patients. Although the participation rate can be considered good, our final sample size is small and patients with the mildest disease course were underrepresented. If the sample had been restricted to either more or less severe cases or those who had suffered from RA shorter or longer periods of time, the findings may have been different. Activities or functional abilities which are considered important in the early stages of disease may become less relevant as the pattern of disease changes [13].

The observed variation in importance scores between the twenty functions included in HAD-DI implies that the functions have an inconsistent role in the RA patient's everyday life. If an overall measure of patients' functional ability is calculated by using equal weights for all functions, as is the case in e.g. HAQ and MHAQ [7, 9], the index figures do not necessarily represent the burden RA causes to the patients. With a small sample of 25 inpatients with relatively severe disease status Hewlett et al. showed that HAQ items do not have universal value [11], which was corroborated by the present study findings. However, the present study finding that 'Taking a tub bath' was considered significantly less important than all other functions, was different from the earlier study, where none of the twenty functions were deemed unimportant [11]. One reason for this may be the difference in patients' disease status, or the fact that during the last decades tub baths have become more and more rare in Finland. 
For MHAQ, Pincus et al. chose intuitively one function from each HAQ-DI domain, instead of the worst ability out of two or three functions used in the original index [9]. Present study findings support the choice they made intuitively earlier. Using 'Wash and dry your body' instead of 'Get on and off the toilet' probably does not limit the coverage of functions in MHAQ as movements included in sitting are included in 'Get in and out of bed' and 'Get in and out of car'. In an earlier study, RA patients with more severe disease status ranked practically the same functions as the most important ones within each of the eight domains [11], further validating the selection of Pincus et al. [9].

Factor analyses formed one distinct factor with high loadings in very different functions. These loadings did not accumulate to any particular HAQ domain, but all domains included functions with high loadings. One interpretation for this could be that most of the functions included in the original HAQ and all those in MHAQ are important components of RA patients' well-being. All eight functions intuitively chosen for MHAQ from the original list of twenty functions for HAQ-DI had high factor loadings. This may be considered as a further indication of rheumatology health professionals' success in their selection process [9]. On the other hand, development of the RecentOnset Arthritis Disability (ROAD) questionnaire showed that soliciting the important functional items from health professionals with varying clinical backgrounds and variety of patients also produced an item pool of much wider variety [17].

Functions related to movements of lower extremities have been suggested to play an important role in RA patients' disability $[18,19]$. However, the ROAD questionnaire development suggested that upper extremity functions explain most of the variance. One reason for their contradictory finding may be the final selection of the items they used $[17,20]$. In the present study, the roles of upper 
and lower extremities were distinguished as entities, as movements related to these activities had high loadings in factor analysis. Overall, the factor loadings may suggest that reduced locomotor activities of the extremities and large body movements are more important than fine movements of the upper extremity.

Perceived importance of the functions was only weakly related to the corresponding perceived functional ability. If the level of disability and perceived importance were strongly correlated, then the level of importance of a function could be considered as a proxy for the level of disability. The variations in these relationships indicate that subjects who experienced higher disability in some function were not necessarily those who considered that function important. Perceived importance was not directed by level of disability, which supports the idea that the assessments quantify independently the relative importance of the functions. However, this is somewhat in contradiction with the earlier suggestion that patient preferences for improvement in health may be an alternative for the assessment of important patient outcomes [16]. The patient's contribution to assessment of health status and treatment outcomes, as well as the magnitude of their role requires further experimentation [21].

Present study findings corroborate the view that assessment of RA patients' functional status would benefit from further development of measures by giving relative weights from patients' perspective to those functional abilities which are used for index computations. 
Acknowledgements

This study has been supported by grants from the research funds of The Hospital District of Southwest Finland and from Wyeth AB. 
Refenrences

1. Pinals R, Masi A, Larsen R (1981) Preliminary criteria for clinical remission in rheumatoid arthritis. Arthritis \& Rheum 24:1308-15.

2. Huskisson E (1974) Measurement of pain. Lancet 2:1127-31.

3. Smedstad L, Kvien T, Moum T, Vaglum P (1997) Correlates of patients' global assessment of arthritis impact. A 2-year study of 216 patients with RA. Scand J Rheumatol 26:259-65.

4. van der Heijde D, van't Hof M, van Riel P, Theunisse L, Lubberts E, van Leeuwen M, van Rijswijk M, van de Putte L (1990) Judging disease activity in clinical practice in rheumatoid arthritis: first step in the development of a disease activity score. Ann Rheum Dis 49:91620.

5. van der Heijde D, van't Hof M, van Riel P, van de Putte L (1993) Development of a disease activity score based on judgment in clinical practice by rheumatologists. J Rheumatol 20:579-81.

6. Prevoo M, van Riel P, van’t Hof M, van Rijswijk M, van Leeuwen M, Kuper H, van de Putte L (1995) Validity and reliability of joint indices. A longitudinal study in patients with recent onset rheumatoid arthritis. Br J Rheumatol 32:589-94.

7. Fries J, Spitz P, Kraines G, Holman H (1980) Measurement of patient outcome in arthritis. Arthritis \& Rheum 23:137-45.

8. Bruce B, Fries J (2005) The Health Assessment Questionnaire (HAQ). Clin Exp Rheumatol 23:14-8.

9. Pincus T, Summey J, Soraci S jr., Wallston K, Hummon N (1983) Assessment of patient satisfaction in activities of daily living using a modified Stanford health assessment questionnaire. Arthritis \& Rheum 26:1346-53. 
10. Wolfe F (2001) Which HAQ is best? A comparison of the HAQ, MHAQ and RA-HAQ, a difficult 8 item HAQ (DHAQ), and a rescored 20 item HAQ (HAQ20): Analyses in 2491 rheumatoid arthritis patients following Leflunomide initaiation. J Rheumatol 28:982-9.

11. Tugwell P, Bombardier C, Buchanan W, Goldsmith C, Grace E, Hanna B (1987) The MACTAR patient preference functional priority approach for assessing improvement in physical disability in clinical trials in rheumatoid arthritis. J Rheumatol 14:446-51.

12. Hewlett S, Smith A, Kirwan J (2001) Values for function in rheumatoid arthritis: patients, professionals, and public. Ann Rheum Dis 60:928-33.

13. Carr A, Hewlett S, Hughes R, Mitchell H, Ryan S, Carr M, Kirwan J (2003) Rheumatology outcomes: The patient's perspective. J Rheumatol 30:880-3.

14. Verhoeven A, Boers M, Linden van der S (2000) Validity of the MACTAR questionnaire as a functional index in a rheumatoid arthritis clinical trial. J Rheumatol 27:2801-9.

15. Tuominen R, Tuominen S, Suominen C, Möttönen T, Azbel M, Hemmilä J. Perceived functional disabilities among rheumatoid arthritis patients. Rheumatol Int 2009 E-publ.

16. Kvien T, Heiberg T (2003) Patient perspective in outcome assesments - perceptions or something else. J Rheumatol 30:873-6.

17. Salaffi F, Bazzichi L, Stancati A, Neri R, Cazzato M, Consensi A, Grassi W, Bombardieri S (2005) Development of a functional disability measurement tool to asses early arthritis: the Recent-Onset Arthritis Disability (Road9 questionnaire. Clin Exp Rheumatol 23:628-36.

18. Pincus T, Sokka T, Kautiainen H (2005) Further development of a physical function scale on a multidimensional health assessment questionnaire for standard care of patients with rheumatoid arthritis. J Rheumatol 32:1432-9.

19. Mason J, Anderson J, Meenan R, Haralson K, Lewis-Steavens D, Kaine J (1992) The rapid assessment of disease activity in rheumatology (radar) questionnaire. Validity and 
sensitivity to change of a patient self-reported measure of joint count and clinical status. Arthritis \& Rheum 35:156-62.

20. Salaffi F, Stancati A, Neri R, Grassi W, Bombardieri S (2005) Measuring functional disability in early rheumatoid arthritis: the validity, reliability and resposiveness of the Recent-Onset Arthritis Disability (ROAD) index. Clin Exp Rheumatol 23(Suppl. 39):S31S42.

21. Kirwan J, Heiberg T, Hewlett S, Hughes R, Kvien T, Ahlmen M, et al. (2003) Outcomes from the patient perspective workshop at OMERACT 6. J Rheumatol 30:868-72. 
Table 1. Median values with Interquartile ranges (IQR) for the perceived importance of the functions frequently asked from RA patients to estimate their functional ability by sex

Function

DRESSING AND GROOMING

Wash your hair

Dress yourself

ARISING

Stand up from a chair

Get in and out of bed

EATING

Cut your meat

Lift a full glass to your mouth

Prepare your own food

WALKING

Walk outdoors on flat ground

Climb five steps
Men IQR

$86.5 \quad 66.2-93.8$

$93.0 \quad 83.8-97.8$

$95.0 \quad 88.0-97.8$

$94.0 \quad 90.3-97.8$

$68.5 \quad 33.5-94.0$

$93.0 \quad 78.8-96.8$

$78.0 \quad 62.8-95.8$

$97.0 \quad 92.5-99.0$

$93.0 \quad 75.5-97.8$

\section{Women IQR}

All IQR

$89.0 \quad 73.5-97.0$

$88.0 \quad 72.3-96.0$

$95.0 \quad 79.5-97.5$

$93.0 \quad 82.3-97.0 \quad$ P H

$94.0 \quad 85.5-97.0$

$95.0 \quad 87.0-97.0$

$95.0 \quad 87.0-97.5$

$95.0 \quad 88.0-97.8$

$\mathrm{PH}$

$61.0 \quad 39.0-88.0$

$58.0 \quad 30.5-89.0$

$90.0 \quad 68.0-96.0$

$91.0 \quad 75.0-96.0 \quad \mathrm{PH}$

$88.0 \quad 71.5-96.0$

$83.5 \quad 62.8-96.0$ 
Table 1. continued

HYGIENE

Wash and dry your body

Take a tub bath

Get on and off the toilet

$\mathrm{REACH}$

Reach and get down a $2 \mathrm{~kg}$ flourbag just above your head

Bend down and pick up clothing from the floor

GRIP

$$
\begin{array}{rr}
94.0 & 91.0-99.0 \\
6.5 & 3.0-82.3 \\
97.5 & 92.8-99.0
\end{array}
$$

$69.0 \quad 23.5-88.3$

$91.0 \quad 76.0-98.0$

$92.0 \quad 61.8-98.0$

$81.5 \quad 51.3-92.3$

$92.0 \quad 84.8-99.0$
$95.0 \quad 87.0-98.5$

$4.0 \quad 2.0-32.0$

$94.0 \quad 90.5-98.0$

$72.0 \quad 43.5-89.5$

$67.5 \quad 34.0-88.8$

$83.0 \quad 64.5-96.0$

$86.0 \quad 64.3-97.8 \quad$ P H

Open jars which have been

previously opened

Turn faucets on and off
$80.0 \quad 53.0-96.5$

$87.0 \quad 55.3-97.0$

$74.0 \quad 51.5-90.0$

$73.5 \quad 50.0-90.0$

$93.0 \quad 66.5-98.0$ $\begin{array}{lll}95.0 & 90.3-99.0 \quad \mathrm{P}\end{array}$

$5.0 \quad 2.3-43.8$

$96.5 \quad 91.0-99.0 \quad \mathrm{H}$ 
Table 1. continued

ACTIVITIES

Run errands and shop

Get in and out of a car

Vacuum

\begin{tabular}{cc}
91.5 & $64.5-98.8$ \\
94.5 & $73.8-98.8$ \\
\hline 85.5 & $54.0-97.5$
\end{tabular}

$87.0 \quad 58.5-96.5$

$90.0 \quad 60.5-97.5$

$65.0 \quad 45.0-96.0$
$88.5 \quad 62.0-97.8$

$93.0 \quad 67.8-98.0 \quad \mathrm{P}$

$70.5 \quad 45.0-95.8$

$\mathrm{P}=$ The function representing the domain in MHAQ by Pincus et al.(9)

$\mathrm{H}=$ The function ranked highest in the domain by Hewlett et al.(11) 
Table 2. Factor loadings of the relative importance scores based on Spearman's rank correlations

One factor model

0.766

0.846

Dress yourself

ARISING

Stand up from a chair

Get in and out of bed

0.788

0.786

EATING

Cut your meat

0.652

Lift a full glass to your mouth

Prepare your own food

WALKING
0.855

Climb five steps
Two factor model

Factor $1 \quad$ Factor 2

$0.697 \quad 0.175$

$\begin{array}{lll}0.872 & -0.051 \quad \mathrm{PH}\end{array}$

$0.874 \quad-0.198$

$\begin{array}{lll}0.876 & -0.209 \quad \mathrm{P} \mathrm{H}\end{array}$

$0.457 \quad 0.516$

$\begin{array}{lll}0.771 & 0.025 & \mathrm{P} \mathrm{H}\end{array}$

$0.478 \quad 0.354$

$0.937 \quad-0.190 \quad \mathrm{PH}$

$0.756 \quad 0.120$ 
Table 2. continued

\section{HYGIENE}

Wash and dry your body

0.861

Take a tub bath

0.004

Get on and off the toilet

0.883

RAECH

Reach and get down a $2 \mathrm{~kg}$ flourbag

just above your head $\quad 0.657$

Bend down and pick up clothing from the floor

0.794

GRIP

Open car doors

0.720

Open jars which have been previously opened

0.644

Turn faucets on and off

0.822

\section{ACTIVITIES}

Run errands and shop

0.646

Get in and out of a car

0.787

Vacuum
0.716

$\begin{array}{lll}0.878 & -0.038 & \mathrm{P} \\ -0.106 & 0.300 & \\ 0.930 & -0.107 & \mathrm{H}\end{array}$

0.415

0.653

0.728

$0.170 \quad \mathrm{P} \mathrm{H}$

$0.663 \quad 0.150$

$0.411 \quad 0.630$

$\begin{array}{lll}0.857 & -0.089 & \text { P H }\end{array}$

$\begin{array}{llll}0.477 & 0.434 & \mathrm{H} \\ 0.715 & 0.183 & \mathrm{P} \\ 0.577 & 0.367 & \end{array}$


Table 2. continued

$\mathrm{P}=$ The function representing the domain in MHAQ by Pincus et al.(9)

$\mathrm{H}=$ The function ranked highest in the domain by Hewlett et al.(11) 